\title{
Scalable Neutron Imaging Systems at Compact Sources
}

\author{
Knud Thomsen*, Eberhard Lehmann, and Markus Strobl \\ Laboratory for Neutron Scattering \& Imaging, Paul Scherrer Institut, CH-5232 Villigen PSI, Switzerland
}

\begin{abstract}
Neutron imaging offers unique capabilities not limited to the structural characterisation of materials, components and processes at a large variety of neutron sources and thus is highly qualified to be a prime candidate for instrumentation at compact neutron sources. The peculiar characteristics of neutrons, and in particular the cross sections for their interaction with matter enable imaging results that are directly comparable but, most notably, are effectively complementary to those of wide spread X-ray imaging characterisation. Here, it is highlighted how neutron imaging can significantly add to the value and the versatility of basically all and especially future compact neutron sources. Diverse advantages are identified at all possible sizes and levels of sophistication of compact sources. These areas range from source characterisation and students training to state of the art neutron imaging in 3D to applications and method development, which are possible at compact sources and some of which can take particular advantage of advanced source characteristics such as e.g. several target stations at a single accelerator.
\end{abstract}

\section{Introduction}

Neutron imaging (NI) offers a number of unique features qualifying it as a prime candidate for instrumentation at compact neutron sources. The peculiar characteristics of neutrons, in particular their interaction cross sections, allow for image contrast that is in principle comparable but, in particular, complementary to that of easily available X-ray imaging. Neutron imaging, initially on film, was first established as a non-destructive testing tool for industrial use, but through digitalisation and ever increasing resolution capabilities quickly developed into a powerful research tool in many fields of applications.

The most advanced NI facilities, defining the state-ofthe-art of the technique, are located at large scale neutron sources where the access routes to beam time are strictly regulated and time consuming.

The establishment of neutron imaging techniques at new compact sources has several key aspects:

- Sustainable in a landscape of a decreasing number of large sources, in particular reactor based

- Potential impact of individual methods to the design and tuning of source properties, which can be optimized according to the different requirements

- Flexibility in the instrument layout (e.g. multiple beams, time structure, variable collimation, sample environment, ...) in order to optimize for method and application

- Easy access to beam time for researchers and industry in particular in the vicinity of the source and in-house, and also for students

\subsection{Aspects Relevant to Compact Neutron Sources}

Technically, the range for possible installations is vast; this likewise holds for price, sophistication and potential use. Neutron imaging is in principle possible at rather small sources yielding only a relatively low neutron flux. However, e.g. a demanding state-of-the-art detection systems (very high sensitivity, long exposure enabled) is a pre-requisite for impactful application. A very instructive example is the $2 \mathrm{~W}$ research reactor of the TU Dresden where imaging is pursued (compare image data in Fig. 1).

At the other end of the scale it is possible to take full advantage of a most versatile compact source concept with several independent target stations, like it is intended e.g. in the High Brilliance Source (HBS) project. In such case one can conceive even unparalleled opportunities e.g. for dynamic neutron imaging, like in the idea of crossed neutron beams for stereoscopy with neutrons. Such option would hardly be accessible at any other type of neutron source. However, such options require detailed consideration concerning applications and corresponding instrumentation by the source designers from the very beginning. This should entail a high priority for neutron imaging as a technique with outstanding potential and capabilities and well suited to be established at such neutron sources.

\subsection{Current situation for Neutron Imaging at existing Compact Source facilities and Projects}

In the overview given in [1] 23 neutron sources are listed and described in some details. From these sources, 11 declare to intend (or perform) some neutron imaging. However, regarding scientific literature or even the

\footnotetext{
* Corresponding author: knud.thomsen $@$ psi.ch
} 
homepages of the facilities only about 6 display relevant and impactful results.

A very positive example in this regard are the Japanese sources which are additionally networked in the JCANS collaboration [2] and provide a valuable platform to even progress imaging methods relevant for the large scale source of JPARC during its construction and commissioning. And such valuable work continues due to beam availability limitations during large scale user operation.

Some of the new projects like HBS Jülich (D), SONATE Saclay (F), projects in Hungary, and LENS (USA) have intentions to establish neutron imaging capabilities. It is of outmost importance, however, that these projects early on gain access to expertise and practical knowledge in the respective technology of neutron imaging and its applications in order to enable optimum design decisions.

\section{Imaging as a Key Application at Sources of Different Power and Various Levels of Sophistication}

Neutron imaging holds the potential to provide several valuable functions for a small scale source project which include self-supporting applications for the project, industrial use cases and science cases of various nature as well as method development. In the following, some key fields of neutron imaging are briefly introduced.

\subsection{Self-supporting Project Applications}

\subsubsection{Source/Moderator Characterisation}

Sources and moderators can be optimized for specific instrumentation and in particular for neutron imaging and even different imaging modalities. Specifically, wavelengths/spectra, sizes, flux, time structure and homogeneity have to be considered and carefully designed. Taking the perspective the other way around, neutron imaging set-ups allow for a detailed characterization, in particular concerning flux, spectrum, divergence and respective homogeneity of emanating neutron beams. Thus pinhole imaging can support efficient facility development and validation of numerical simulation tools. In particular small sources can provide significant flexibility for ongoing optimization of the source layout, and will hence especially provide from such assessment capability.

\subsubsection{Training and Preparation Capabilities}

Advantageous educational aspects are a direct consequence of these manifold possibilities: students can easily become acquainted with neutron-based instrumentation, make their first steps and can almost immediately obtain useful and illustrative results.

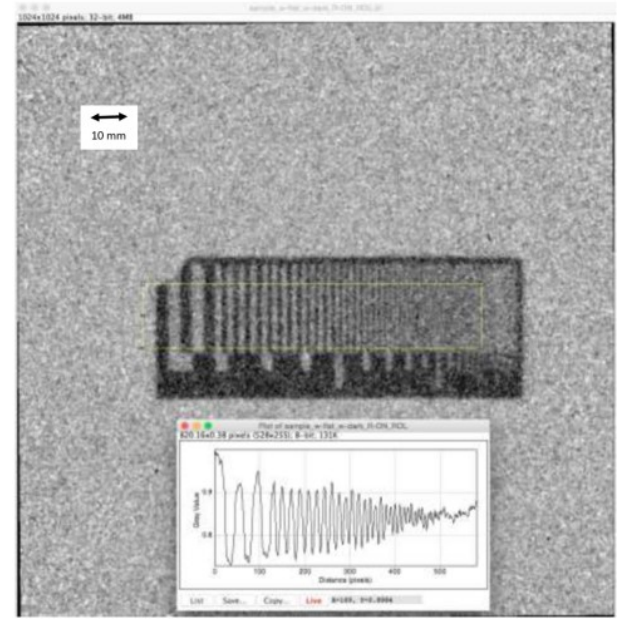

Fig. 1. Example image obtained at $8 \times 10^{3} \mathrm{n} / \mathrm{cm}^{2} \mathrm{~s}$ in $30 \mathrm{~min}$ (AKR-2, TU Dresden); image courtesy C. Lange, TU Dresden, Germany

Access at a small source is inherently easier than at big facilities, and hands-on experiments are possible, which cannot be done at more powerful installations. This, in turn allows for a "pathfinder" function where experiments are tried and optimized before expensive beam time at a big source is applied for. As a proof of principle, simple neutron radiography can yield valuable images at fluxes as low as $10^{4} \mathrm{n} / \mathrm{cm}^{2}$ s (see Fig. 1). In parallel to increasing possibilities at larger and more complex, still compact, neutron sources, preparatory experiments can be performed before going to flagship neutron facilities with optimized set-ups.

\subsubsection{Public Relations Capabilities}

The results of neutron imaging are particularly well suited to provide insights to and generate understanding in the general public for neutrons and neutron science, thus supporting the project and facility. Likewise results and valuable science or industrial applications directly accessible through the observation of high quality images are also exceptionally well suited to generate impact at funding bodies and governance and thus ensure sustainability.

\subsection{Industrial Uses}

Neutron imaging can provide information of interest to diverse industry and cover a vast range of questions. Quality Assurance topics, in particular improving processing, quality and reliability of products can be claimed to lie at a centre of gravity of relevant investigations in industry. Neutrons deliver especially valuable results e.g. for pyrotechnic components, joining parts, comprising welding, soldering/brazing and, in particular, adhesive bonding (see Fig. 2) but also e.g. processing such as sintering. 


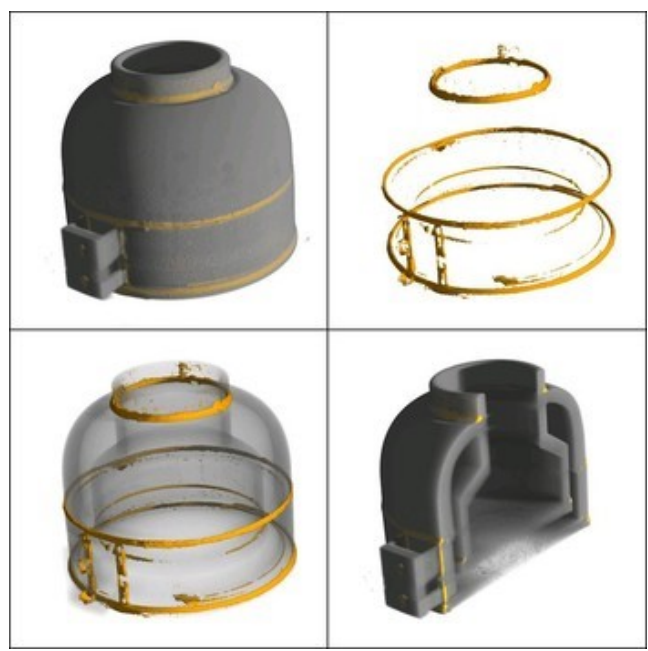

Fig. 2. Example image of assessment of soldering quality, semi-transparent 3D representation highlighting the Boron containing brazing solder distribution.

Further examples include humidity distributions in building materials, but also investigations on the effect of storage on syringes containing medicine, rocket [3] and automotive components, to name but a few.

A specific example of an application at a small scale source is provided in Fig. 3. It concerns a relatively new field, which is to assess relevant characteristics of specimen produced by metal additive manufacturing.

Novel manufacturing technologies like additive ones are often used for sophisticated and expensive parts; typical areas include aerospace and medical industries. For these, special requirements apply also with respect to quality control. Neutron imaging is a tool of choice for such applications.

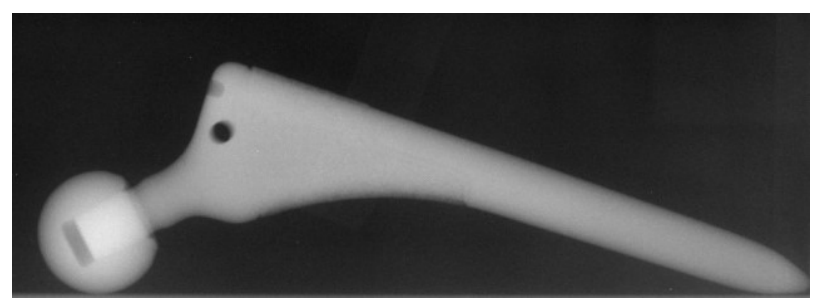

Fig. 3. Example of image of hip replacement, obtained with a moderated $14 \mathrm{MeV}$ D-T source, image courtesy M. Taylor, Phoenix Lab, Madison, USA.

Figure 3 shows the result obtained using a small commercial neutron source, which employs the D-T reaction. Neutrons are moderated and 3 hours of exposure at a thermal flux of approximately $1 \times 10^{4}$ $\mathrm{n} / \mathrm{cm}^{2} \mathrm{~s}$ were required to obtain the presented result.

\subsection{Science Cases}

2.3.1. Engineering materials research

Additive manufacturing is also a dominating topic in engineering materials research and neutron imaging is utilized as post processing characterisation tool supporting process optimisation and simulation of metallic materials and components. The main focus is on bulk metallic materials, which cannot be penetrated by other radiation for imaging purposes, and the achieved porosity and porosity distribution, dependent on process parameters. Novel imaging methods are also used to map strains and phases in the built material, which bear key aspects of mechanical behaviour of the material, material strength and durability under service conditions.

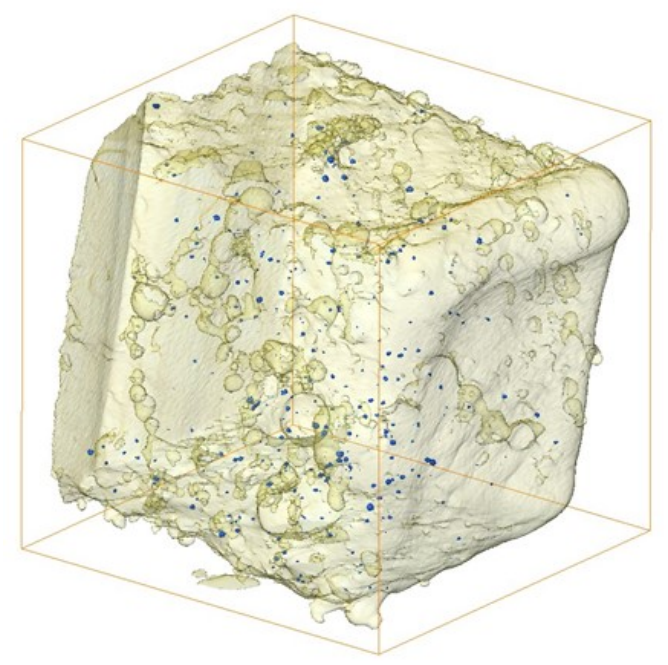

Fig. 4. Example image of porosity in additively manufactured gold (courtesy R. Loge, EPFL) at the sub-10 $\mu \mathrm{m}$ scale obtained with the PSI neutron microscope detector (illustrating a far aim for compact sources) [4]

Figure 4 displays a specific example targeting the porosity in additive manufacturing of precious metals, which is of interest for jewellery and watch industries.

Other key topics concern welding, phase transformations and strain in steels or magnetic domain structures in transformer steels as well as hydrogen and hydrogen embrittlement in metals, corrosion and many others more.

\subsubsection{Energy Research}

The specific high sensitivity of neutrons for some light elements, namely hydrogen and lithium, make neutrons and especially neutron imaging an outstanding research and development tool in the key field energy conversion techniques especially with respect to fuel cells, hydrogen storage and batteries, but also nuclear safety. These also spur industrial interest and applications and examples are numerous in recent literature [5-13].

Many of these investigations are also suited for small sources, in particular in observations of steady states, which can be done in their own right but also establish ideal preparation work for extensions to in operando characterisations.

\subsubsection{Soil, Plants and Environment}

Recent literature also underlines the distinguished capabilities of neutron imaging in the field of geology, soil and plants and particular the interplay of the latter in the context of water balance and uptake. 
Due to the fact that neutron imaging is an excellent tool for the observation of water and liquid flow in porous media a large variety of geological and agriculturally relevant processes and phenomena can be observed with high spatial and time resolution, and especially outstanding means for quantification of related transport phenomena.

\subsubsection{Cultural Heritage}

Unexpected images of familiar as well as of unknown objects like deciphering the internal make-up of, e.g., ancient sacred statues are considered interesting by most people and important to those researching ancient culture and technology (Fig 5).

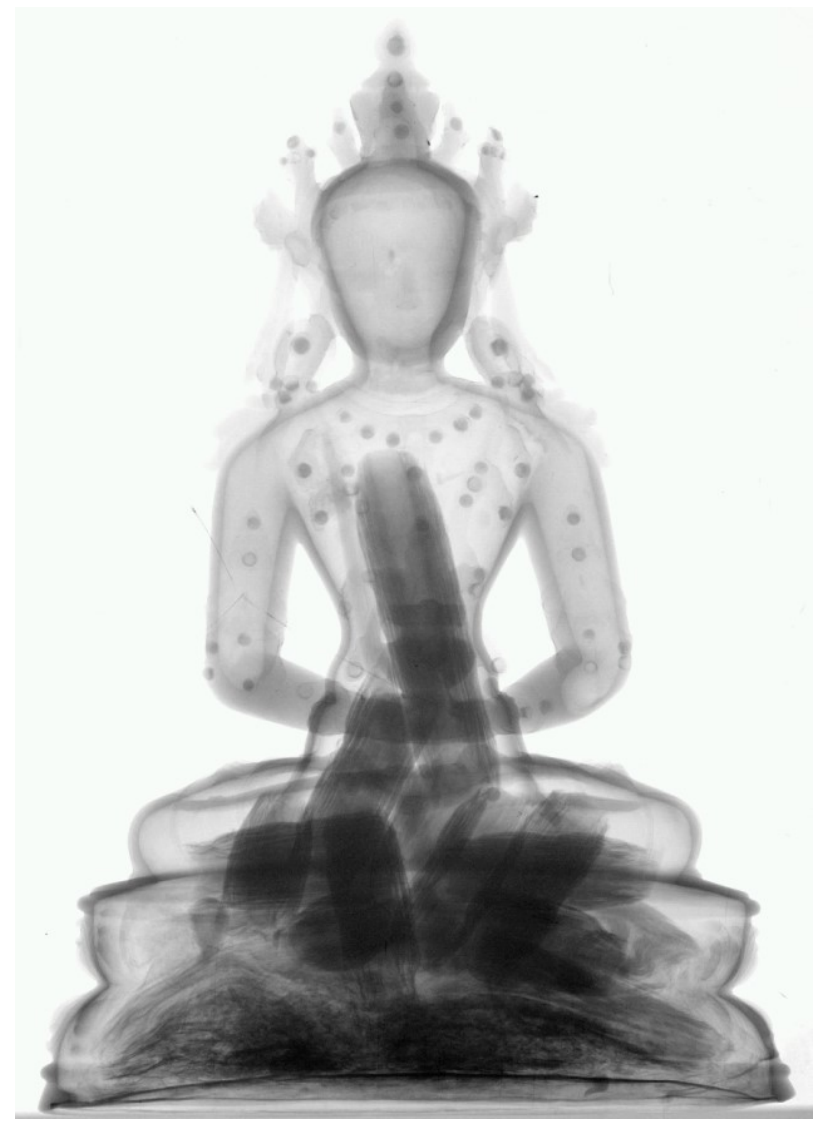

Fig. 5. Example for cultural heritage studies: neutron image of a Tibetan Buddha from the $15^{\text {th }}$ century, showing the filling with scrolls

Neutron imaging is an invaluable tool for the investigation of invaluable cultural heritage as it allows to gain insights in a non-destructive manner, which is often the only possibility to investigate unique and valuable artefacts in details and thus provide unique insights into our past and origin.

It is very often such applications that offer a unique way to demonstrate to the common taxpayer as well as to funding agencies the high potential and manifold usefulness of neutron-based techniques starting at flux levels as low as $1000 \mathrm{n} / \mathrm{cm}^{2} \mathrm{~s}$.

\subsection{Development of new Methods}

The development of novel methods at dedicated small sources, potentially even featuring multiple targets and moderators offers an open-ended avenue. Advantages with respect to method development are (i) better availability of beamtime for tests and experiments, (ii) more flexible instrumentation, potentially (iii) down to the neutron source itself.

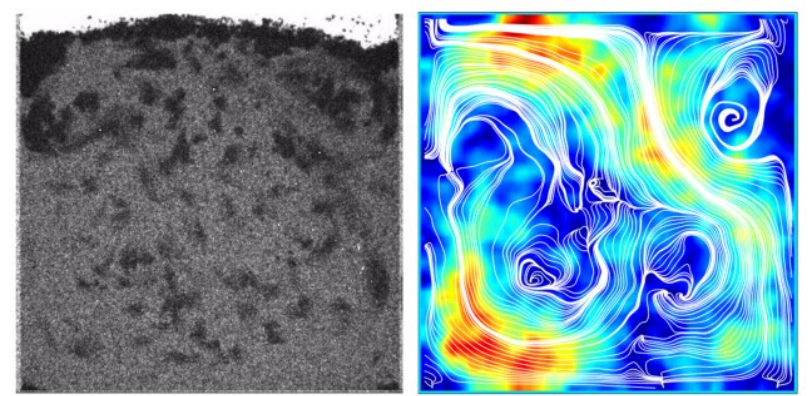

Fig. 6. Example image of recent application aimed at improving imaging- as well as simulation-approaches: moving inclusions in electromagnetically agitated liquid metal, comparison image obtained with neutrons versus fluid dynamic numerical simulation [5]

Outstanding examples are the developments for time-offlight imaging at JPARC performed at HUNS in Hokkaido, including sophisticated techniques like polarized neutron imaging [15]. Another example might be the test beamline for ESS in Berlin supporting the development of a sophisticated wavelength frame multiplication chopper system not only for neutron imaging [16]. Despite the neutron source BERII being a medium flux reactor source, with regards to time-offlight through mimicking the ESS time structure, it can be considered a small source in this context.

Some small sources might also provide so far unparalleled opportunities for new concepts and ideas to be tested and realized. A small source with several target stations might for example enable to cross neutron beams from different targets at a sample position. This might provide the combination of different spectra, time structures or techniques. An example in neutron imaging might be found related to recent studies at PSI investigating the movement of inclusions in liquid metal as relevant for industrial manufacturing processes (Fig. 6) and required for the development of reliable simulation tools [5]. The challenge to observe the process with $3 \mathrm{D}$ spatial resolution, despite the speed of the process which does not enable tomographic scans, could be solved through neutron stereoscopy utilizing crossed neutron beams.

\section{General Considerations \& Conclusion}

Neutron Imaging is a non-invasive inspection technique with high relevance in several areas of research and industry.

Based on modern digital detection techniques and numerous methodical developments in the past years it has become attractive for research fields like e.g. electrochemistry, engineering materials, energy applications, construction materials, geology and also archaeometry. 
On the other hand, industry can take profit directly in the investigations of advanced manufacturing processes and products, as well as in R\&D activities like in the past for Diesel particular filters but also representative inspections like e.g. of adhesive joints, to highlight some prominent examples only.

Nevertheless, state-of-the-art neutron imaging is currently established only at distinct places, where strong neutron sources and dedicated beam lines are available. A list of the neutron imaging installations operated in a user lab are provided in [17], but about 100 facilities used around the globe are attempting to apply neutron imaging [18].

Reasons for the currently low exploitation of this rewarding technique might include:

- $\quad$ Strong neutron sources have been built in the past dedicated to different use cases: nuclear fuel development (irradiation experiments), isotope production, neutron scattering, nuclear engineering education and neutron activation analysis.

- most neutron sources are not mobile or even portable (like X-ray sources) and the inspection of specimen has to be performed "on-site" with often restricted access for important stakeholders of neutron imaging (limiting also awareness)

- neutron scattering communities with coherent applications and purely academic intentions have been dominating the design and applications of research neutron sources for the past decade

- the neutron imaging user community is highly diverse and thus less suited to effectively lobby for their needs

- $\quad$ modern neutron imaging with digital detectors and capable of quantitative also scientific studies emerged delayed to diffraction and spectroscopy techniques

The approach of building compact and tuneable neutron sources offers new challenges in efficient exploitation also for the NI community. It is an outstanding opportunity to build dedicated imaging stations, optimized from the cradle to the grave from the initial source to the detector. At most sources so far, in contrast, a compromise has to be found between the different instruments and stakeholders in the design and particularly the neutronic design of the source.

Today thermal and cold neutron spectra are mainly used for state-of-the-art NI, while fast neutrons are utilized only in few cases and for special purposes. While highflux pulsed neutron beams are only available at very few powerful spallation sources (JPARC, ISIS, IBR-2, ...) time-of-flight (TOF) methods at continuous sources entail significant flux penalties.

However, very sensitive neutron imaging detectors are meanwhile available and in operation which enable working also with low beam intensities and provide neutron images with reasonable image quality. The combination of tuneable neutron sources with the most suitable and technologically advanced detection system is set to enable valuable and impactful NI also beyond the traditional settings of large-scale facilities.

Aspects that require special (re-)consideration concerning imaging at small sources include:

- all nuclear reactions initially provide high energy neutrons first (on the order of some $\mathrm{MeV}$ ) and moderation reduces the available flux significantly; can and how can fast neutrons be used efficiently for highly relevant neutron imaging applications?

- what is the optimum source and moderator for specific relevant neutron imaging techniques and can their parameters be tuned even supplying an individual beamline?

- what are the requirements to the time structure and pulsed beam flux to be relevant for advanced ToF imaging?

- $\quad$ are there relevant advanced techniques and in particular imaging methods and applications that can profit from novel concepts such as crossed beams from multiple target stations realized at a single accelerator (compare HBS project [19])?

- while current neutron imaging detector technique serves well the current state of the art in neutron imaging, novel sources and instruments still provide challenges for efficient utilisation. What specific demands are expected in particular from future small neutron sources?

For the moment there are only very few suppliers of compact sources on the market. Some test facilities have been built and upgraded and a few new installations appear to be planned and to advance. None of them is optimized for the exclusive and dominant use for neutron imaging. However, informed design work and applied expertise in neutron imaging are needed to build the best options and to identify challenging applications and use cases matching the particular conditions.

\section{References}

1. I. Anderson et al., Research opportunities with compact accelerator driven sources, Physics Report 654, 1-58 (2016)

2. Y. Kiyanagi, Neutron Imaging at Compact Accelerator-Driven Neutron Sources in Japan, J. Imaging, 4, 55; doi:10.3390/jimaging4040055 (2018)

3. G. Bayon, Review on use of neutron radiography at Saclay nuclear research center, Proc. $3^{\text {rd }}$ World Conference on Neutron Radiography, pp. 439-446 (1996)

4. V. Manzi-Orezzoli et al., Coating Distribution Analysis on Gas Diffusion Layers for Polymer Electrolyte Fuel Cells by Neutron and X-Ray High 
Resolution Tomography, ACS Omega, ID: ao-201901763j.R2 (2019)

5. M. Ščepanskis et al., Assessment of Electromagnetic Stirrer Agitated Liquid Metal Flows by Dynamic Neutron Radiography, Metallurgical and Materials Transactions B ISSN 1073-5615 Volume 48 Number 2 Metall and Material Trans B 48:10451054 DOI 10.1007/s11663-016-0902-8 (2017)

6. M. Siegwart, et al., Selective Visualization of Water in Fuel Cell Gas Diffusion Layers with Neutron Dark-Field Imaging Journal of The Electrochemical Society 166(2):F149-F157 (2019)

7. M. Makowska, et al., Phase transition mapping by means of neutron imaging in SOFC anode supports during reduction under applied stress, ECS Transactions, 68 (1) 1103-1114 (2015)

8. I. Manke, et al., Characterization of water exchange and two-phase flow in porous gas diffusion materials by H-D contrast neutron radiography, Appl. Phys. Lett. 92, 244101 (2008)

9. M. Strobl, et al., Topical Review: Advances in neutron radiography and tomography, J. Phys. D 42 243001 (2009)

10. Thomas Knoche et al., In situ visualization of the electrolyte solvent filling process by neutron radiography, Journal of Power Sources 331, 267276 (2016)

11. Y. Wu et al., Effect of serpentine flow-field design on the water management of polymer electrolyte fuel cells: An in-operando neutron radiography study, Journal of Power Sources 399, 254 (2018)
12. M. Cochet et al., Novel Concept for Evaporative Cooling of Fuel Cells: an Experimental Study Based on Neutron Imaging, Fuel Cells 18, 619 (2018)

13. P. Stahl et al., An Investigation of PEFC Sub-Zero Startup: Influence of Initial Conditions and Residual Water, Fuel Cells 17, 778 (2017)

14. P. Boillat, E.H. Lehmann, P. Trtik, M. Cochet Neutron imaging of fuel cells ? Recent trends and future prospects, Current Opinion in Electrochemistry 5, 3 (2017)

15. M. Strobl, H. Heimonen, S. Schmidt, M. Sales, N. Kardjilov, A. Hilger, I. Manke, T. Shinohara, J. Valsecchi Topical review: Polarisation measurements in neutron imaging J. Physics D 52, 12 (2019)

16. M. Strobl, M. Bulat, K. Habicht, The wavelength frame multiplication chopper system for an ESS test-beamline and corresponding implications for ESS instruments, Nucl. Instr. Meth. A 705, 74-84 (2013)

17. E. H. Lehmann, D. Ridikas, Status of Neutron Imaging - Activities in a Worldwide Context, Physics Procedia Volume 69, 2015, 10-17 (2015)

18. http://isnr.de/index.php/facilities/facilities-worlwide

19. HBS Project, Conceptual Design Report, FZ Jülich, ISBN 978-3-95806-280-1 (2017) 